\title{
The role of LRRK2 in inflammatory bowel disease
}

\author{
Zhihua Liu' ${ }^{1}$, Michael J Lenardo ${ }^{2}$ \\ ${ }^{1}$ Institute of Biophysics, Chinese Academy of Sciences, Beijing 100101, China; ${ }^{2}$ Laboratory of Immunology, National Institute of \\ Allergy and Infectious Diseases, National Institutes of Health, Bethesda, MD 20892-1892, USA \\ Cell Research (2012) 22:1092-1094. doi:10.1038/cr.2012.42; published online 20 March 2012
}

\begin{abstract}
$L R R K 2$ was recently identified as a major susceptibility gene for Crohn's disease (CD) by genome-wide association studies (GWAS). LRRK2 deficiency in mice confers enhanced susceptibility to experimental colitis. In this research highlight, we discuss the current understanding of LRRK2 in the context of colitis, and postulate the future directions.
\end{abstract}

Inflammatory bowel disease (IBD) is believed to result from inappropriate and sustained host immune responses to intestinal microbiota. IBD is a so-called "complex" disease, which is influenced by multiple environmental factors and genetic factors with each of them playing partial roles. Like other complex diseases, the unbiased appraisal of the human genome by genome-wide association studies (GWAS) has been particularly successful in identifying genes and genetic loci that show significant association with IBD. To date, close to 100 genes or genetic susceptibility loci have been identified $[1,2]$. In-depth studies on the IBD susceptibility genes have pointed to specific pathways involving intestinal homeostasis, microbial recognition, reactive oxygen species (ROS) generation, autophagy, and immune regulation. In this research highlight, we will discuss our current understanding

Correspondence: Michael J Lenardo

Tel: +1-301-496-6754; Fax: +1-301-402-8530

E-mail: lenardo@nih.gov of the role of LRRK2 in IBD.

LRRK2 is a complex protein with 2527 amino acids, containing a RAS of complex GTPase domain (ROC), a C-terminal of ROC domain (COR), and a Ser/Thr kinase domain (Figure 1A), in addition to three potential protein interaction domains: an ankyrin domain (ANK), leucine-rich repeats (LRR), and WD40 repeats. The involvement of the $L R R K 2$ locus in CD was first suggested by a meta-GWAS study in 2008 [3]. This finding was further confirmed by two other meta-GWAS studies $[1,2]$.

Besides IBD, LRRK2 is better known for its role in familial Parkinson's disease (PD) [4]. Substantial investigation has been carried out to understand the physiological role of LRRK2 and the pathogenicity of those mutations. A number of biological functions have been ascribed to LRRK2, ranging from regulating protein translation through phosphorylating E4-BP, controlling microRNA-mediated translational repression, contributing to neurite extension through interaction with Rac1, regulating autophagy by some unknown mechanism, accelerating neurite degeneration induced by $\alpha$-synuclein [4]. However, it is still debated whether kinase activity of LRRK2 is essential for its involvement in PD. Furthermore, the holy grail of the research of LRRK2 in $\mathrm{PD}$, the physiological function of LRRK2 and its role in neuronal pathology in PD, is still highly contested.

In immune cells, LRRK2 expression is largely restricted to myeloid cells and B cells $[5,6]$. The expression profile of LRRK2 suggests that an experimental colitis model, such as dextran sodium sulfate (DSS)-induced colitis, in which innate immune cells play a major role in the pathogenesis of the disease, would be a useful tool to study the function of LRRK2. Compared with wild-type mice, LRRK2-deficient mice are more susceptible to DSS-induced colitis, as manifested by rapid weight loss, worse clinical symptoms, and more severe colon pathology (Figure 1B). This observation suggests that LRRK2 may negatively regulate inflammation.

How does LRRK2 deficiency account for exacerbated DSS-induced colitis? The complex nature of the multidomain LRRK2 protein makes it plausible that LRRK2 may regulate different pathways in immune reactions. For instance, it has been found that LRRK2 associates with the phagosomal membrane once a bacterium such as Salmonella is phagocytozed by a murine macrophage cell line RAW [6], though the physiological role of this association remains to be explored. Furthermore, it has been found that LRRK2 regulates ROS production in both the neuronal cell line SH-SY5Y and macrophage cell line RAW through undetermined mechanism $[4,6]$. One interesting finding comes from a high-throughput RNAi screen in Drosophila S2R+ cells. dLRRK, the LRRK2 Drosophila homolog, turns out to be a regulator of NFAT1 in 

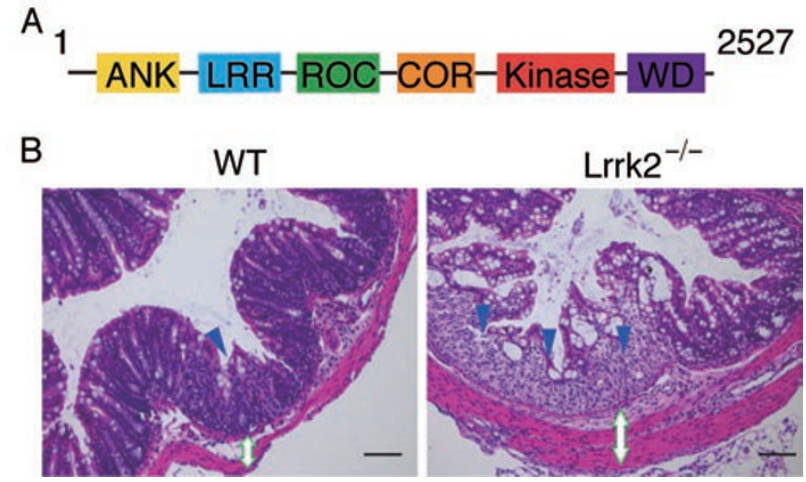

Figure 1 (A) A schematic diagram of LRRK2. (B) The worsen colitis in Lrrk2 ${ }^{-1-}$ mice treated with DSS. Figure 1B was adapted from Liu et al. [13].

a NFAT1-GFP-based screening [7]. Indeed, both in vitro and in vivo evidence suggest that the mammalian LRRK2 inhibits NFAT1 in macrophages.

NFAT1 is one of the key regulators involved in regulating immune responses [8]. NFAT1 is highly phosphorylated and resides in the cytoplasm. Upon $\mathrm{Ca}^{2+}$ influx, NFAT1 is dephosphorylated by calcineurin, translocates to nucleus, and promotes transcription of its target genes. Besides this central paradigm, there is a RNA-protein complex controlling NFAT1 nuclear translocation in a manner that has not been fully understood [9]. NFAT1 is well recognized for its role in IL-2 production in T cells [8]. Recently, the role of NFAT1 in myeloid cells is beginning to be appreciated. In dendritic cells (DC) and macrophages, NFAT1 has been noted for controlling the production of a range of cytokines, including IL-2, IL-6 and TNF- $\alpha$. NFAT1 has been reported to promote inflammatory cytokine production in $\mathrm{T}$ cells in IBD murine model and in human CD patients $[10,11]$; however, whether the activity of NFAT 1 functions in immune cells other than $\mathrm{T}$ cells has not been well-studied in IBD.

Detailed biochemical studies have found that LRRK2 regulates NFAT1 by participating in the NRON complex, and does not affect NFAT1 phosphorylation directly or indirectly [9]. Willingham and colleagues previously identified a non-coding RNA, NRON, as a NFAT suppressor through a siRNA-based screen using a human non-coding RNA library [9]. Further investigation has found that NRON and a number of proteins assemble into a large complex, called the NRON complex, which regulates NFAT nuclear translocation. Notably, some Drosophila homologs of protein components of the NRON complex, including Cul4B, KPNB1 and CSE1L, have been identified independently as the regulators for NFAT in the same siRNA screen in Drosophila $\mathrm{S} 2 \mathrm{R}+$ cells, which identified dLRRK [7]. A study of another protein in the complex, IQGAP1, which does not have a Drosophila homolog, has revealed that IQGAP1, in synergy with NRON, potentiates NFAT activation [12]. The fact that LRRK2 physically interacts with multiple components of the NRON complex and the functional evidence that the inhibitory effect of LRRK2 on NFAT1 depends on NRON suggests that LRRK2 is a major component of the NRON complex [13].

What makes LRRK2 an interesting constituent of the NRON complex is that LRRK2 is subjected to lipopolysaccharide (LPS)-induced degradation. The exact mechanism by which the NRON complex suppresses NFAT1 remains to be determined. LPS, a common TLR4 ligand for activating macrophages and dendritic cells, triggers a rapid degrada- tion of LRRK2, which may help release NFAT1 from the NRON complex and promote NFAT1 nuclear translocation. This phenomenon may provide an interesting insight that NFAT1 activation can be modulated by other signals in addition to the essential $\mathrm{Ca}^{2+}$ influx.

But how is the observation that LRRK2 deficiency in mouse exacerbates DSS-induced colitis related to IBD in humans? The majority of the SNPs on LRRK2 associated with IBD are located in non-coding intronic regions, except for rs376186. What effect could those SNPs have on the level of LRRK2? Previous studies on SNPs associated with IBD have shown that they cause either qualitative or quantitative changes. For example, the common exonic synonymous SNP (rs 10065172) on IRGM abrogates the regulation by a family of microRNAs, which leads to quantitative changes in protein levels [14]. Another SNP (rs2066847) found at the NOD2 locus leads to an early stop codon, generating a truncated protein with altered biochemical properties [15]. While the effect, if any, of the SNPs in the noncoding region of LRRK2 remains to be determined, the high-risk allele of rs376186 decreases LRRK2 stability, which may lead to a lower LRRK2 level in its carriers. Whether this lower level of LRRK2 in Met2397 carriers explains their increased risk of IBD needs to be further verified in large-scale human studies. Furthermore, whether the other SNPs on LRRK2 cause similar changes in LRRK2 levels remains to be determined.

Currently, we are still at the beginning of understanding the role of LRRK2 in IBD and immune regulation. Many questions are left unanswered. For example, what is the function of LRRK2 in B cells? Is LRRK2 expressed in other cell types, such as neutrophils? How does LRRK2 in B cells and other cells contribute to IBD? On the other hand, among the many interesting findings regarding LRRK2 in PD, do any of them apply to the function of LRRK2 in 
immune cells? Furthermore, the role of NFAT in myeloid cells in autoimmune diseases, such as IBD, has not been thoroughly investigated. NFAT has been previously reported to promote both inflammatory and anti-inflammatory cytokine production, therefore, the physiological role of NFAT activity may depend on the specific pathogenic conditions.

In brief, GWAS have provided important insights into genetic contributions to complex diseases. An important goal is to translate these genetic connections to a physiological and pathological understanding of each individual gene into the etiology of the disease. Knowledge gained from specific genedeficient mice has contributed important understanding to IBD pathogenesis in humans. Therefore, further studies on LRRK2-deficient mice or -transgenic mice may provide more insights into the role of LRRK2 in IBD and immune responses.

\section{Acknowledgments}

This work is supported by the Intramural Research Program of the NIH, NIAID, and NIA. We thank C Kanellopoulou and HB Cai for suggestions and comments.

\section{References}

1 Anderson CA, Boucher G, Lees CW, et al. Meta-analysis identifies 29 additional ulcerative colitis risk loci, increasing the number of confirmed associations to 47. Nat Genet 2011; 43:246-252.

2 Franke A, McGovern DP, Barrett JC, et al. Genome-wide meta-analysis increases to 71 the number of confirmed Crohn's disease susceptibility loci. Nat Genet 2010; 42:1118-1125.

3 Barrett JC, Hansoul S, Nicolae DL, et al. Genome-wide association defines more than 30 distinct susceptibility loci for Crohn's disease. Nat Genet 2008; 40:955-962.

4 Lin CH, Tsai PI, Wu RM, Chien CT. LRRK2 Parkinson's disease: from animal models to cellular mechanisms. Rev Neurosci 2011; 22:411-418.

5 Hakimi M, Selvanantham T, Swinton $\mathrm{E}$, et al. Parkinson's disease-linked LRRK2 is expressed in circulating and tissue immune cells and upregulated following recognition of microbial structures. J Neural Transm 2011; 118:795-808.

6 Gardet A, Benita Y, Li C, et al. LRRK2 is involved in the IFN-gamma response and host response to pathogens. $J$ Immunol 2010; 185:5577-5585.

7 Gwack Y, Sharma S, Nardone J, et al. A genome-wide Drosophila RNAi screen identifies DYRK-family kinases as regulators of NFAT. Nature 2006; 441:646-650.

8 Hogan PG, Chen L, Nardone J, Rao A. Transcriptional regulation by calcium, calcineurin, and NFAT. Genes Dev 2003; 17:2205-2232.

9 Willingham AT, Orth AP, Batalov S, et al. A strategy for probing the function of noncoding RNAs finds a repressor of NFAT. Science 2005; 309:1570-1573.

10 Weigmann B, Lehr HA, Yancopoulos $\mathrm{G}$, et al. The transcription factor NFATc 2 controls IL-6-dependent T cell activation in experimental colitis. $J$ Exp Med 2008; 205:2099-2110.

11 Shih TC, Hsieh SY, Hsieh YY, et al. Aberrant activation of nuclear factor of activated $\mathrm{T}$ cell 2 in lamina propria mononuclear cells in ulcerative colitis. World J Gastroenterol 2008; 14:17591767.

12 Sharma S, Findlay GM, Bandukwala HS, et al. Dephosphorylation of the nuclear factor of activated T cells (NFAT) transcription factor is regulated by an RNA-protein scaffold complex. Proc Natl Acad Sci USA 2011; 108:1138111386.

13 Liu Z, Lee J, Krummey S, Lu W, Cai $\mathrm{H}$, Lenardo MJ. The kinase LRRK2 is a regulator of the transcription factor NFAT that modulates the severity of inflammatory bowel disease. Nat Immunol 2011; 12:1063-1070.

14 Brest P, Lapaquette P, Souidi M, et al. A synonymous variant in IRGM alters a binding site for miR-196 and causes deregulation of IRGM-dependent xenophagy in Crohn's disease. Nat Genet 2011; 43:242-245.

15 Strober W, Kitani A, Fuss I, Asano $\mathrm{N}$, Watanabe T. The molecular basis of NOD2 susceptibility mutations in Crohn's disease. Mucosal Immunol 2008; 1 Suppl 1:S5-9. 